\begin{tabular}{|c|l|}
\hline Title & On ageneralization of the Constantin_Lax-Majda equation \\
\hline Author(s) & Okamoto, Hisashi; Sakajo, Takashi; Wunsch, Marcus \\
\hline Citation & $\begin{array}{l}\text { Nonlinearity, 21(10), 2447-2461 } \\
\text { https://doi.org/L0.1088/0951-7715/21/10/013 }\end{array}$ \\
\hline Issue Date & 2008_10 \\
\hline Doc URL & http://hdl.handle.net/2115/34775 \\
\hline Rights & ○ 2008IOP Publishing \\
\hline Type & article (author version) \\
\hline File Information & CLM_Nonlinearity_final.pdf \\
\hline
\end{tabular}

Instructions for use 


\title{
On a generalization of the Constantin-Lax-Majda equation
}

\author{
Hisashi Okamoto ${ }^{1}$, Takashi Sakajo ${ }^{2}$ and Marcus Wunsch ${ }^{3}$ \\ ${ }^{1}$ Research Institute for Mathematical Sciences, Kyoto University, Kyoto 606-8502 \\ Japan. Partially supported by JSPS Grants 17204008 and 20244006 \\ E-mail: okamoto@kurims.kyoto-u.ac.jp \\ 2 Department of Mathematics, Hokkaido University, Sapporo 060-0810, Japan. \\ Partially supported by JSPS Grants 17684002 and 19654014. PRESTO, Japan \\ Science Technology Agency. \\ E-mail: sakajo@math.sci.hokudai.ac.jp \\ ${ }^{3}$ Faculty of Mathematics, University of Vienna, Nordbergstrasse 15, 1090 Wien, \\ Austria. He expresses his gratitude for the support of the ministry of Education, \\ Culture, Sports, Science and Technology of Japan.
}

E-mail: marcus.wunsch@univie.ac.at

\begin{abstract}
We present evidence on global existence of solutions of De Gregorio's equation, based on numerical computation and a mathematical criterion analogous to the Beale-Kato-Majda theorem. Its meaning in the context of a generalized ConstantinLax-Majda equation will be discussed. We then argue that a convection term, if set in a proper form and in a proper magnitude, can deplete solutions of blow-up.
\end{abstract}

AMS classification scheme numbers: 35Q35, 76B03

Submitted to: Nonlinearity 


\section{Introduction}

De Gregorio [7, 8] proposed the following differential equation as a model of 3D vorticity dynamics of incompressible inviscid fluid flow:

$$
\omega_{t}+v \omega_{x}-v_{x} \omega=0
$$

where $\omega$ is the unknown function representing the strength of the vorticity, and $v$ is determined by $v_{x}=H \omega$ with $H$ being the Hilbert transform. In this paper we consider the equation (1) in $-\pi<x<\pi$ with the periodic boundary condition. Therefore, $H \omega$ and $v$ are given as

$$
H \omega(t, x)=\frac{1}{2 \pi} \int_{-\pi}^{\pi} \omega(t, y) \cot \left(\frac{x-y}{2}\right) \mathrm{d} y
$$

where $\int$ implies Cauchy's principal value, and

$$
v(t, x)=\frac{1}{\pi} \int_{-\pi}^{\pi} \omega(t, y) \log \left|\sin \frac{x-y}{2}\right| \mathrm{d} y,
$$

respectively. It is also easy to see that $v=-\left(-\frac{\mathrm{d}^{2}}{\mathrm{~d} x^{2}}\right)^{-1 / 2} \omega$.

We first give numerical evidence which shows that the solution of (1) exists globally in time. De Gregorio [7, 8] considered (1) in order to contrast it with

$$
\omega_{t}-v_{x} \omega=0, \quad v_{x}=H \omega .
$$

This equation is called the Constantin-Lax-Majda equation (CLM for short) and was introduced in [6] as a model for blow-up dynamics of vorticity of incompressible inviscid fluid flow. In fact, as is rigorously proved in [6], most of the solutions of (2) blow up in finite time. De Gregorio proposed his equation to show that his equation, though it differs from the CLM equation only by the convection term $v \omega_{x}$, is likely to admit no blow-up. He gave some evidence but mathematical proof is yet to be given, and there is much room for scrutiny. We cannot prove the global existence of solutions of (1), either, but we present accurate numerical results conforming with the global existence.

We then consider a generalization of the CLM equation and De Gregorio's equation in the following form:

$$
\omega_{t}+a v \omega_{x}-v_{x} \omega=0, \quad v_{x}=H \omega,
$$

where $a$ is a real parameter. If $a=0$, it becomes the CLM equation [6]. If $a=1$, it is De Gregorio's equation. If $a=-1$, then this is the equation considered by Córdoba et al. $[4,5]$. The authors of $[4,5]$ considered

$$
\theta_{t}+\theta_{x} H \theta=0
$$

and mathematically proved that this equation possesses many blow-up solutions. If we differentiate (4) and set $\omega=-\theta_{x}$, then $\omega$ satisfies the generalized De Gregorio equation with $a=-1$. Since we are going to argue that the equation (3) with $a=1$ admits no blow-up, this contrast may be of some interest. 
The present paper is organized as follows. A motivation for (3) is explained in Section 2. Section 3 introduces theorems on local existence and a criterion on global existence. Based on these theorems, we give in Section 4 the results by numerical experiments about De Gregorio's equation. Proofs of the theorems are presented in Section 5. Then in Section 6, we prove that the equation (3) in the limit of $a \rightarrow \infty$ admits no blow-up. Concluding remarks are given in Section 7.

\section{The role of the convection term}

It is rather interesting to note the fact that

- the equation (3) with $a=-1$ has blow-up solutions $([4,5])$;

- if $a=0$, most solutions blow up in finite time $([6])$;

- if $a=1$, solutions exist globally in time, which is conjectured in $[7,8]$ and the present paper.

This naturally leads us to the question about which values of $a$ yield global existence for the respective solution.

By analogy with the 3D Euler equations, the term $v \omega_{x}$ in (1) or (3) may be called a convection term. The term $-v_{x} \omega$ may be called a stretching term. In fluid dynamics literature, blow-up of the solutions of the 3D Euler equations is said to be caused by the stretching term. It is also said that the convection term is a kind of neutral player, having little influence on blow-up phenomena. Recently, however, [16] and [17] showed, with many examples, that a convection term often plays a role more important than is usually imagined. Hou and $\mathrm{Li}$ [9] have drawn a similar conclusion for axisymmetric flows with swirl reduced from the 3D Euler and Navier Stokes equations. In fact, blowups can be suppressed by a convection term, if its strength relative to the stretching term (i.e., the modulus of the ratio of their coefficients) is great enough. Accordingly, the determination of blow-up/global-existence would be an interesting problem for (3). We naturally expect that solutions of (3) exist globally in time if $|a|$ is large, and that blow-up is expected if $|a|$ is small. This, however, is a speculation, and rigorous justification is yet to be obtained. It is also worthwhile to see the importance of the sign of $a$. Convection term with $a=-1$ leads us to blow-up, while $a=1$ leads us to global existence. In view of the $3 \mathrm{D}$ vorticity equation, $a=1$ would be better suited to vorticity dynamics model. In fact, on constructing the one-dimensional model equation (3) with $a=1$, De Gregorio [7] introduced the convection term $v_{x} \omega$ so that the relation $v_{x}=H \omega$ between $v$ and $\omega$ represents an one-dimensional analogue of the Biot-Savart formula, which recovers the velocity field from the vorticity field. For this reason, the positive convection term is a natural choice for the one-dimensional model for the threedimensional Euler equations. We are therefore not saying that any form of convection term guarantees global existence. In fact, as was pointed out in [16], an unphysical convection term cannot prevent blow-ups. 
In the present paper, as a first step towards the substantiation of the statement above, we prove in Section 6 that the global existence is guaranteed in the case of $a \rightarrow \infty$, the precise meaning of which will be given later.

It could be helpful to the reader if we here compare the equation (3) with other, similar but different equations. They possess nonlocal nonlinear terms which are different from those in (3).

Morlet [14] considered

$$
\theta_{t}+\delta \theta v_{x}+v \theta_{x}=0, \quad v=H \theta
$$

with $0 \leq \delta \leq 1$. The order of differentiation for $v$ is different from (3). This equation reduces to (4) if $\delta=0$. She proved blow-up of solutions when $0<\delta<1 / 3, \delta=1 / 2, \delta=$ 1. Later Chae et al. [3] proved blow-up for all $0<\delta \leq 1$.

The equation

$$
u_{t}+f u_{x}-a f_{x} u=0, \quad u=-f_{x x}
$$

was considered in [17] and was named the generalized Proudman-Johnson equation. One of its merits is the fact that the equation reduces to the Burgers and Hunter-Saxton equation, for $a=-3$ and $a=-2$, respectively, and it represents similarity solutions of the $m$-dimensional Euler flows for $a=-(m-3) /(m-1)$ for $m=2,3, \cdots$. Blow-up was proved for $a<-1$, and for $-1 \leq a<1$ global existence was proved (see [15]). For $1<a$, the global well-posedness is yet to be settled, but numerical computations strongly suggest blow-up. Thus, it is partly verified that smallness of the stretching term (i.e., $-a f_{x} u$ ) implies global existence.

\section{Local existence and blow-up criterion}

Note first that any solution of (3) satisfies

$$
\frac{\mathrm{d}}{\mathrm{d} t} \int_{-\pi}^{\pi} \omega(t, x) \mathrm{d} x=\int_{-\pi}^{\pi}\left(-a v \omega_{x}+v_{x} \omega\right) \mathrm{d} x=(a+1) \int_{-\pi}^{\pi} v_{x} \omega \mathrm{d} x=(a+1)(H \omega, \omega),
$$

where $(\cdot, \cdot)$ denotes the $L^{2}$ inner-product. Since $H$ is a skew-symmetric operator, we see that $\int_{-\pi}^{\pi} \omega(t, x) \mathrm{d} x$ is independent of $t$. We may therefore specify any value of $\int_{-\pi}^{\pi} \omega(0, x) \mathrm{d} x$. In the present paper, we consider the case where $\int_{-\pi}^{\pi} \omega(0, x) \mathrm{d} x=0$. Accordingly, we use the following function spaces:

$$
\begin{gathered}
L^{2}\left(S^{1}\right) / \mathbb{R}=\left\{f \mid f \in L^{2}(-\pi, \pi), \quad \int_{-\pi}^{\pi} f(x) \mathrm{d} x=0\right\}, \\
H^{k}\left(S^{1}\right) / \mathbb{R}=\left\{f \mid f=\sum_{n=1}^{\infty}\left(a_{n} \cos n x+b_{n} \sin n x\right), \quad \sum_{n=1}^{\infty}\left(a_{n}^{2}+b_{n}^{2}\right) n^{2 k}<\infty\right\},
\end{gathered}
$$

where $k$ is a positive integer. Here, $S^{1}$ denotes the unit circle in the plane. In what follows, it is sometimes regarded as the interval $[-\pi, \pi]$ with $-\pi$ and $\pi$ being identified. 
The symbol $/ \mathbb{R}$ implies that functions with zero mean are collected. A function $\omega(t, \cdot)$ with a frozen $t$ is henceforth denoted by $\omega(t)$. The $L^{2}$ and $L^{\infty}$ norms are denoted by \|\| and \|\|$_{\infty}$, respectively.

The existence local-in-time is guaranteed by the following theorem:

Theorem 3.1 Let $a \in \mathbb{R}$ be given. For all $\omega_{0} \in H^{1}\left(S^{1}\right) / \mathbb{R}$, there exists a $T>$ 0 depending only on $a$ and $\left\|\omega_{0, x}\right\|$ such that there exists a unique solution $\omega \in$ $C^{0}\left([0, T] ; H^{1}\left(S^{1}\right) / \mathbb{R}\right) \cap C^{1}\left([0, T] ; L^{2}\left(S^{1}\right) / \mathbb{R}\right)$ of $(3)$ with $\omega(0)=\omega_{0}$.

The following theorem, which is an analogue of the Beale-Kato-Majda theorem for the 3D Euler equations [2], will later play a crucial role.

Theorem 3.2 Suppose that $\omega(0) \in H^{1}\left(S^{1}\right) / \mathbb{R}$, that the solution of $(3)$ exists in $[0, T)$, and that

$$
\int_{0}^{T}\|H \omega(t)\|_{\infty} \mathrm{d} t<\infty
$$

Then the solution exists in $0 \leq t \leq T+\delta$ for some $\delta>0$.

The proofs of these theorems will be given in section 5 . The criterion (5) will be used in the next section to discuss the global existence of solutions of De Gregorio's equation.

\section{Numerical evidence on the global existence}

In this section, we consider only the case of $a=1$.

Note first that the Hilbert transform is an isometry: $\|H f\|=\|f\|$ for all $f \in L^{2}\left(S^{1}\right) / \mathbb{R}$. Note also that

$$
\|f\|_{\infty} \leq c_{0}\left\|f_{x}\right\| \quad\left(f \in H^{1}\left(S^{1}\right) / \mathbb{R}\right)
$$

with $c_{0}=\frac{\pi}{\sqrt{6}}$. This inequality can be proved easily by the Fourier expansion and the identity $\frac{\pi^{2}}{6}=\sum_{n=1}^{\infty} n^{-2}$.

Since $\|H \omega\|_{\infty} \leq c_{0}\left\|H \omega_{x}\right\|=c_{0}\left\|\omega_{x}\right\|$, Theorem 3.2 implies that no blow-up occurs if $\left\|\omega_{x}(t)\right\|$ remains bounded. In fact, our numerical experiments below suggest that for all $T>0$

$$
\sup _{0 \leq t \leq T}\left\|\omega_{x}(t)\right\|<\infty
$$

Although this is much stronger than the criterion (5), our computations seem to support it. We tried hard to prove mathematically the boundedness of $\left\|\omega_{x}(t)\right\|$ or $(5)$, but we are unsuccessful so far mainly due to the difficulty in handling the Hilbert transform.

Thus, in order to confirm the criterion, we resort to numerical computation. Numerical investigation of the equation (1) was done with the pseudo-spectral method in [18], whose computation showed that $\left\|\omega_{x x}(t)\right\|_{\infty}$ grows very rapidly in finite time. However, the number of modes in the Fourier representation of the solution was 1024, and this might be insufficient for concluding blow-up or global existence. Here, we perform the numerical computation of the equation (1) more accurately, and discuss, 
in a more precise manner, whether the seemingly singular behavior is really a blow-up phenomenon or not.

Our numerical method is the same as that described in [18]: we represent the solution as

$$
\omega(t, x)=\sum_{n=-N / 2}^{N / 2-1} w_{n}(t) \exp (\mathrm{i} n x) \quad(-\pi \leq x \leq \pi)
$$

with $N=16384=2^{14}$. In order to delete the aliasing error, we use the $\frac{2}{3}$-rule, whence we compute the evolution of $w_{n}(t)$ for $|N| \leq 5000$. As the temporal integration, we use the fourth-order Runge-Kutta method with the step size $\Delta t=0.001$. In the course of our experiments, we found spurious growth of the round-off error in high frequency modes. We therefore adopt a spectral filtering technique (see [12]) in which we set the Fourier modes that are smaller than the prescribed threshold value $1.0 \times 10^{-12}$ to zero at every time step so that we avoid the spurious growth of the round-off error in numerical solutions. In what follows, we assume that the initial data are odd functions of $x$, i.e. $w_{n}(0)+w_{-n}(0)=0$. Then, since it is easy to see that the solution is odd in $x$ for all time, we have only to track the evolution of $w_{n}(t)$ for $n=1, \cdots, 5000$. Furthermore, we also assume that the initial data should have at least two non-zero modes in the Fourier representation, since, as is noted in [7] $\omega(t, x)=A \sin k x$ for arbitrary $A \in \mathbb{R}$ and an integer $k$ is a stationary solution of (1).

We first investigate the solutions for the following initial data

$$
\omega_{0}(x)=\sin x+\epsilon \sin 2 x, \quad \epsilon>0 .
$$

Figure 1 shows the numerical results for $\epsilon=0.1$ in $0 \leq t \leq 7.0$. While $\omega(t, x)$ seems to be smooth for all time, a thin spine appears in the first derivative and the second derivative grows rapidly at around $x=0$. From Figure $1(\mathrm{~d})$ the reader might imagine that the solution blows up in finite time. However, Figure 1(b) and 1(c) seem to indicate $\|H \omega(t)\|_{\infty} \leq c\left\|H \omega_{0}\right\|_{\infty}$ and $\left\|\omega_{x}(t)\right\| \leq c\left\|\omega_{0, x}\right\|$, respectively, where the constant $c$ is the unity or very close to the unity. If this is the case for all $t$, then Theorem 3.2 guarantees global existence.

Next, in order to see the singular behavior more closely, we look at the evolution of the magnitude of the spectra $\left|w_{n}(t)\right|$, which is shown in Figure 2. For large $t$, the low-mode spectra are subject to a power-law, whereas the high-mode spectra decay rapidly. In order to study the distribution of spectra quantitatively, let us assume that they behave as

$$
\left|w_{n}\right| \sim C n^{-p} \exp (-\delta n)
$$

for some positive constants $C, \delta$ and $p$. Then we compute the constants by the least square method. The fitting functions approximate the distributions of the spectra accurately as we can see in Figure 2. Figure 3(a) shows the log plot of $\delta(t)$, which indicates a decay exponential in time. This strongly suggests that the solution is smooth for all time. On the other hand, the power $p(t)$ in Figure 3(b) which is shown in 
(a) $\omega(t, x)$

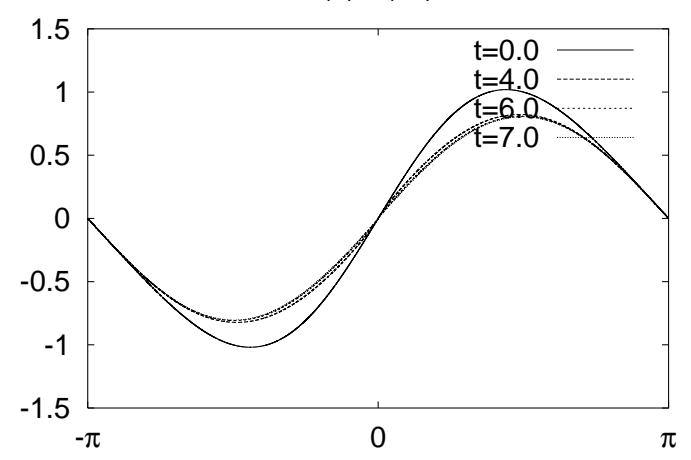

(b) $H \omega(t, x)$

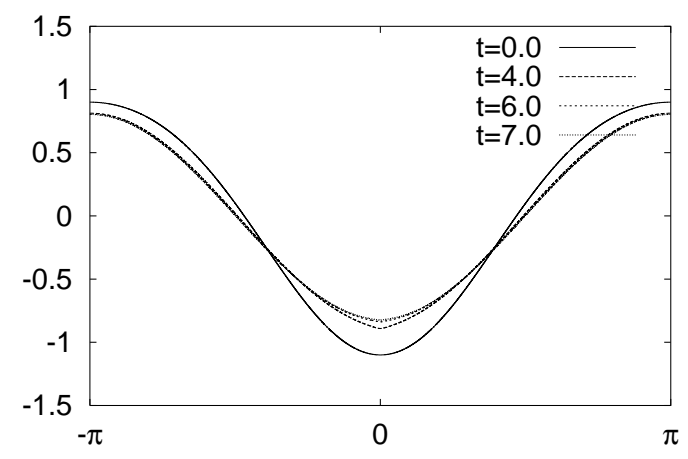

(c) $\omega_{x}(t, x)$

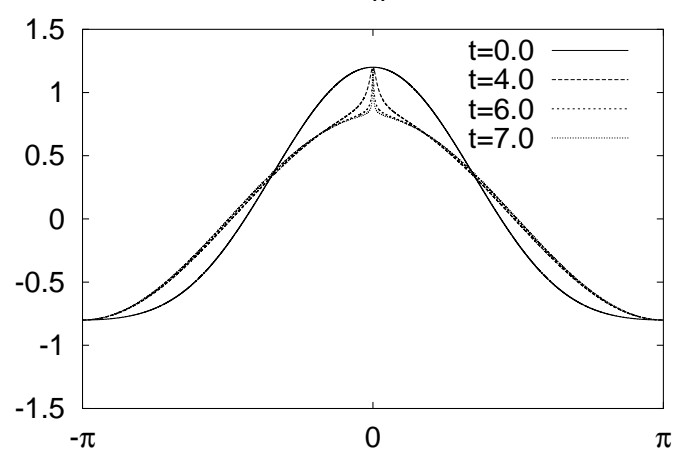

(d) $\omega_{x x}(t, x)$

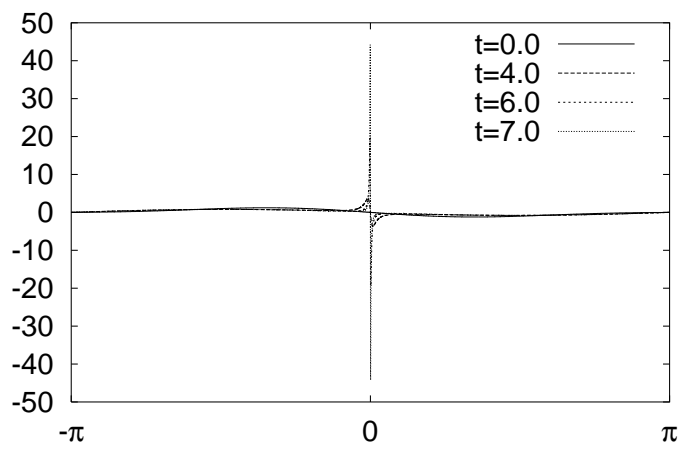

Figure 1. Numerical solution of the equation (1) for the initial data (8) with $\epsilon=0.1$. (a) $\omega(t, x),\left(\right.$ b) $H \omega(t, x)$, (c) $\omega_{x}(t, x)$ and (d) $\omega_{x x}(t, x)$

Figure 3(b) decreases monotonically. We are, however, unable to see its asymptotic value from the numerical data up to this time. We need to compute the solution for longer time to determine it, but the actual numerical computation becomes extremely difficult as $\delta(t)$ gets smaller for large $t$. This is because when $\delta(t)$ is small, the distribution of higher-mode spectra approaches to a power-law and thus the solution cannot be resolved accurately even by 5000 modes.

The exponential decay of $\delta(t)$ is observed in numerical solutions for other initial data, too. Figure 4 shows log plots of $\delta(t)$ computed from the numerical solutions for initial data (8) for $\epsilon=0.2,0.4, \cdots, 1.0$. They show the exponential decay of $\delta(t)$, which 


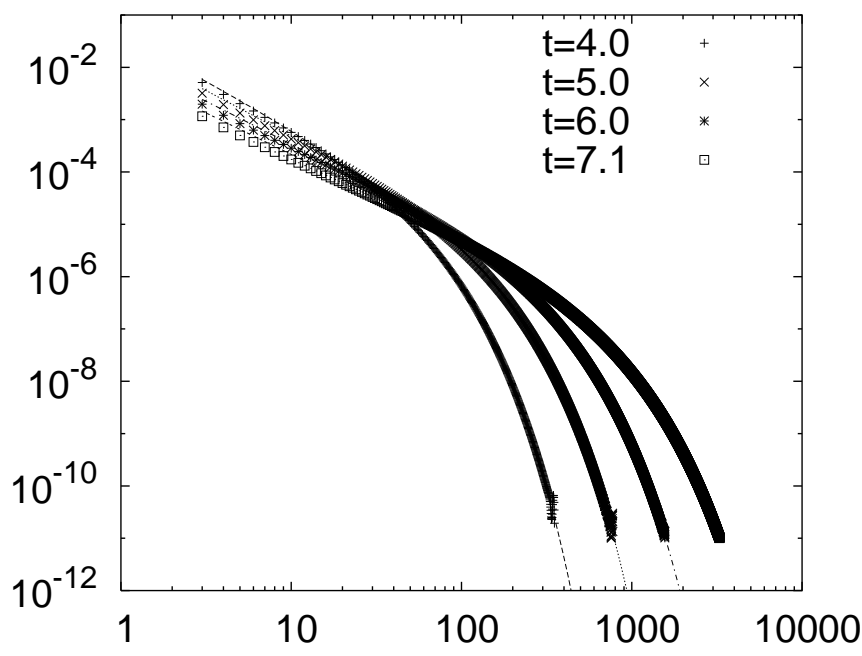

Figure 2. Evolution of the spectra $\left|w_{n}(t)\right|$ of the solution and their approximation function obtained with the least square fit to the Ansatz (9).
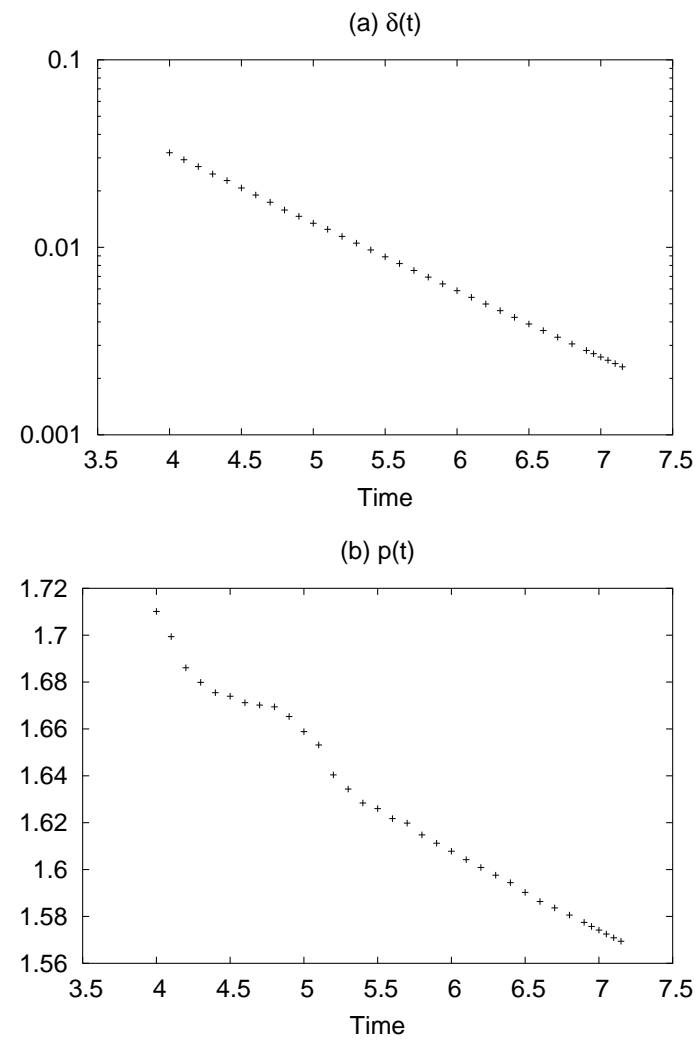

Figure 3. (a) Log plot of $\delta(t)$, (b) plot of $p(t)$ in the Ansatz (9) obtained from the numerical solution. 
conforms with the hypothesis that the solutions are smooth for all time. We show in Figure $5 \omega_{x}(x, t)$ for the initial data with $\epsilon=0.2,0.4,0.6$, and 0.8 , which indicates that $\left\|\omega_{x}(t)\right\|_{\infty} \leq\left\|\omega_{0}\right\|_{\infty}$ up to this time, although $\omega_{x}(t, x)$ acquires a very sharp spine at $x \approx 0$ as $t$ increases. Thus the numerical results verify the condition (7).

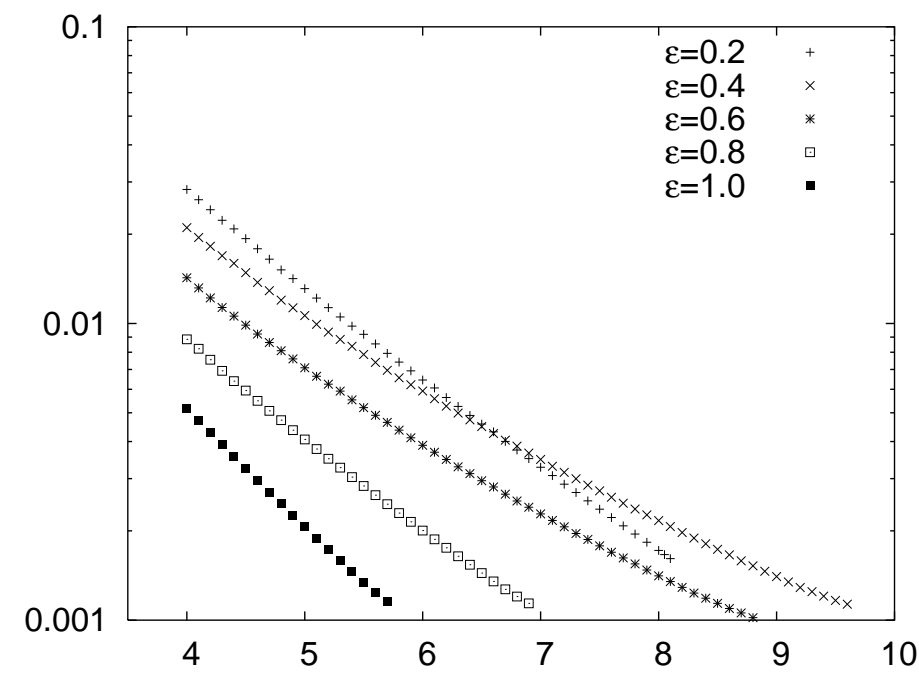

Figure 4. Log plots of $\delta(t)$ for the initial data (8) with various $\epsilon$.

We add some numerical examples to see (7) for other initial data, which are given by

$$
\omega_{0}(x)=\sin m x+0.1 \sin n x,
$$

for various integers $m$ and $n$. Figure 6 shows the evolutions of $\omega_{x}(t, x)$ for $(m, n)=(1,3)$, $(1,4),(2,3)$, and $(2,4)$, which endorses $(7)$ in all the cases. We remark that it is difficult to investigate the distribution of spectra in these cases since the spectra oscillate rapidly so that the least square fit cannot approximate it accurately.

We have thus two ways of supporting the global existence: by Theorem 3.2 and by the positivity of $\delta(t)$.

We finally show another sample computation of (1) with $\omega_{0}(x)=0.2 \cos x+\sin 4 x+$ $\sin 7 x$. The difference of this initial data and those in the previous paragraphs and [18] is that the solutions in [18] are odd functions of $x$, while the present one is not. Figure 7 (a), which was computed with a rather small number - 1024 - of Fourier modes, shows the graph of $\|H \omega(t)\|_{\infty}$, and Figure $7(\mathrm{~b})$ shows that of $\left\|\omega_{x x}(t)\right\|$. They are depicted in the same time interval. Nevertheless, while the rapid increase of $\left\|\omega_{x x}(t)\right\|$ is remarkable, $\|H \omega(t)\|_{\infty}$ seems to remain bounded in the sense of (5).

Summing up these computations, we may well expect that solutions of De Gregorio's equation exist globally in time. This conclusion is reached under the assumption that the numerical computation is accurate and the numerical examples shown here are typical. In order to make a mathematical conclusion, we must prove the criterion (5). But this is difficult for us. 
(a) $\varepsilon=0.2$

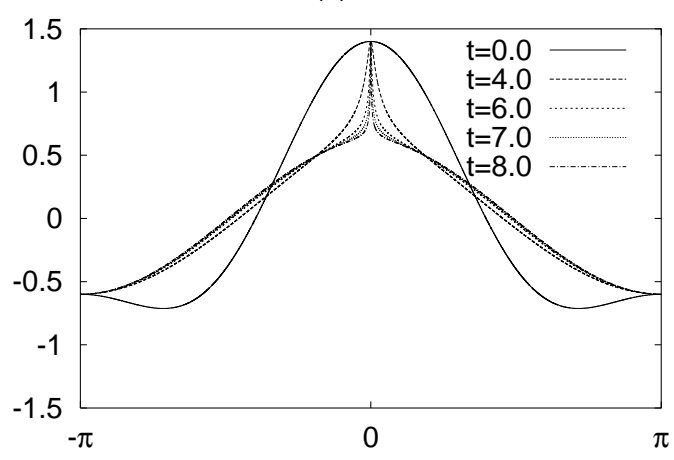

(c) $\varepsilon=0.6$

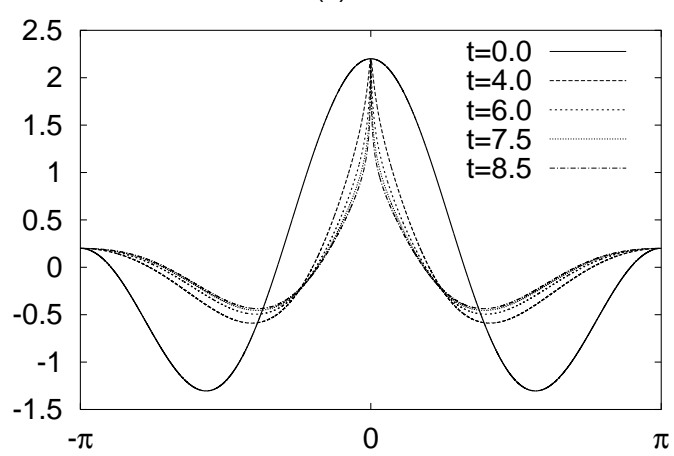

(b) $\varepsilon=0.4$

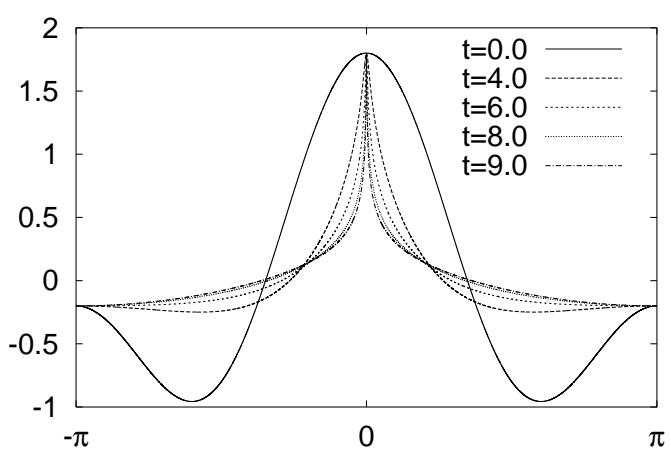

(d) $\varepsilon=0.8$

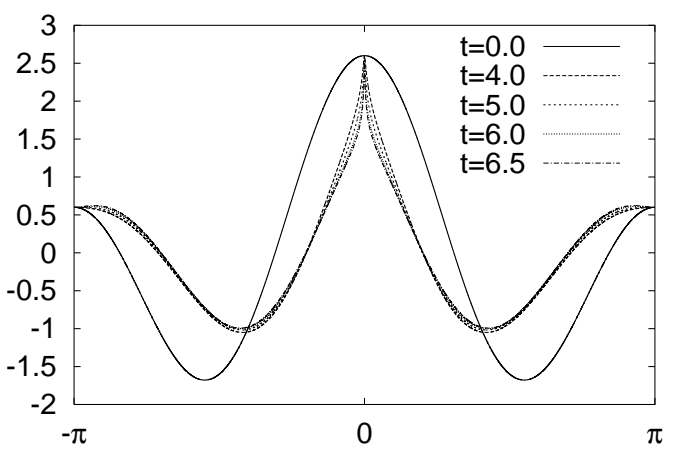

Figure 5. Evolutions of $\omega_{x}(t, x)$ for the initial data (8) with $\epsilon=0.2,0.4,0.6$ and 0.8 .

The reader might wonder whether it is possible that the solution exists in $0 \leq t<$ $\infty$, but it loses the $H^{2}$-smoothness in the sense that $\left\|\omega_{x x}(t)\right\| \rightarrow \infty$ as $t$ approaches a finite $T$. This is actually not the case. The proof of this fact will be given in the next section.

\section{Proofs of Theorems}

In order to prove local existence for (3), we use the following theorem, which is a special case of a theorem by Kato and Lai [10]: Let $V=H^{2}\left(S^{1}\right) / \mathbb{R}, W=H^{1}\left(S^{1}\right) / \mathbb{R}$, and $X=L^{2}\left(S^{1}\right) / \mathbb{R}$. The $L^{2}$ inner-product is denoted by $($,$) . W$ is regarded as a Hilbert space with $\left(f_{x}, g_{x}\right)$ as the inner-product. Similarly, $V$ is equipped with the inner-product $\left(f_{x x}, g_{x x}\right)$. A bilinear form $\langle\rangle:, V \times X \rightarrow \mathbb{R}$ is defined by

$$
\langle f, g\rangle=-\int_{-\pi}^{\pi} f_{x x} g \mathrm{~d} x .
$$

It is then easy to see that

$$
\langle f, g\rangle=\left(f_{x}, g_{x}\right) \quad(f \in V, \quad g \in W) .
$$

Now Kato and Lai's theorem reads as follows: 
(a) $(m, n)=(1,3)$

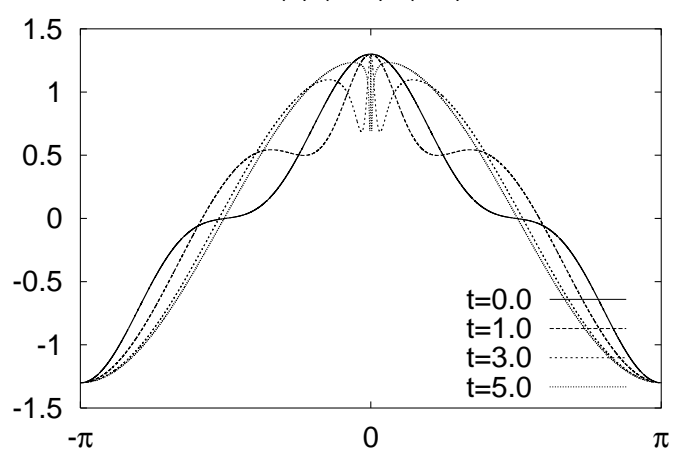

(c) $(m, n)=(2,3)$

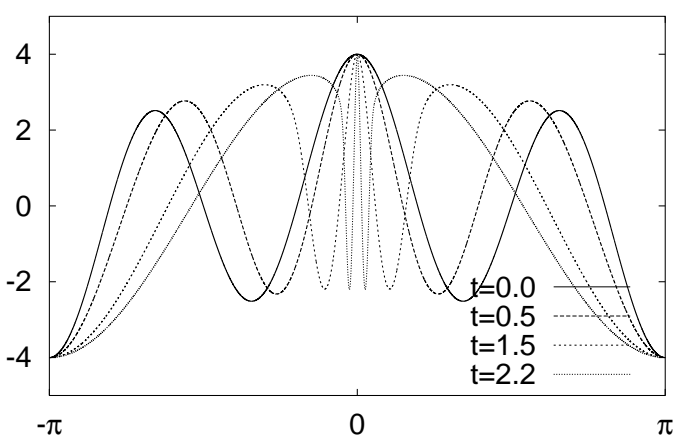

(b) $(m, n)=(1,4)$

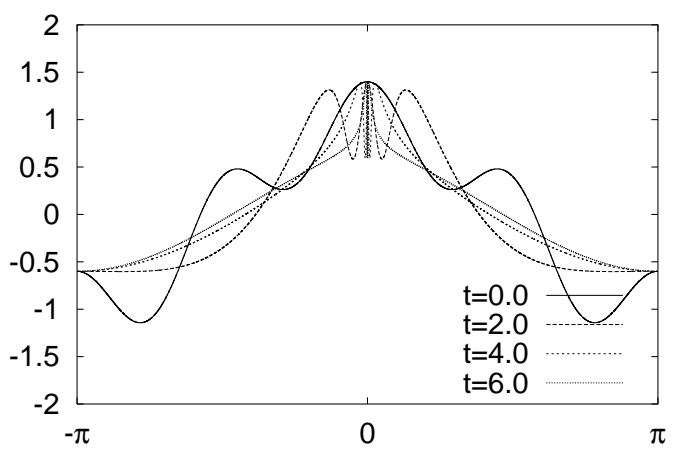

(d) $(m, n)=(2,4)$

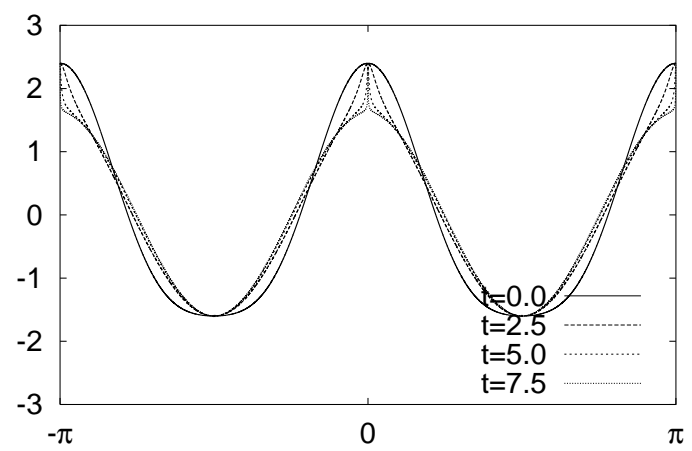

Figure 6. Evolutions of $\omega_{x}(t, x)$ for the initial data (10) with (a) $(m, n)=(1,3)$, (b) $(m, n)=(1,4),(\mathrm{c})(m, n)=(2,3)$ and $(\mathrm{d})(m, n)=(2,4)$.

(a) $\|\mathrm{H} \omega(\mathrm{t})\|_{\circ}$

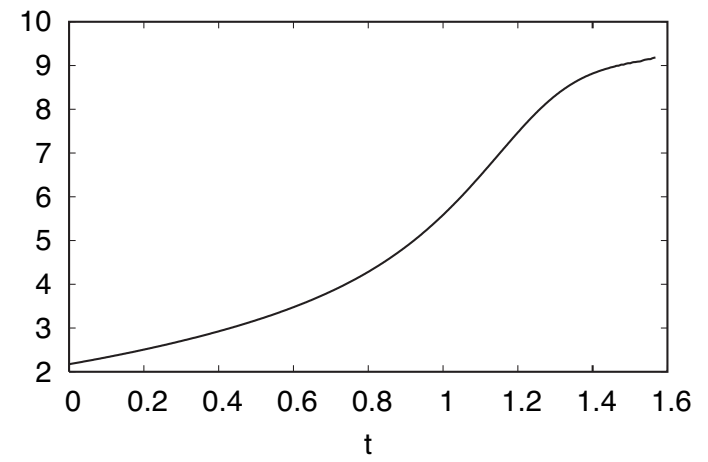

(b) $\left\|\omega_{x x}(t)\right\|$

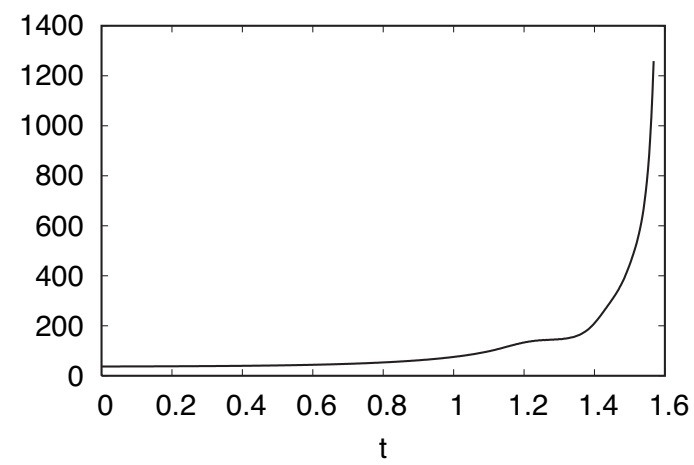

Figure 7. Graphs of $\|H \omega(t)\|_{\infty}$ (a) and $\left\|\omega_{x x}(t)\right\|$ (b). The initial value is $\omega(0, x)=$ $0.2 \cos x+\sin 4 x+\sin 7 x$. 
Theorem 5.1 Suppose that there exists a continuous, nondegenerate bilinear form on $V \times X$, denoted by $\langle$,$\rangle , such that$

$$
\langle v, u\rangle=(v, u)_{W} \quad(v \in V, u \in W)
$$

where $(\cdot, \cdot)_{W}$ denotes the inner-product of $W$. Let $A$ be a sequentially weakly continuous mapping from $W$ into $X$ such that

$$
\langle v, A(v)\rangle \geq-\beta\left(\|v\|_{W}^{2}\right) \quad(\text { for } v \in V),
$$

where $\beta(r) \geq 0$ is a monotone increasing function of $r \geq 0$. Then for any $u_{0} \in W$ there exists $a T>0$ and a solution of $u_{t}+A(u)=0$ and $u(0)=u_{0}$ in the class

$$
C_{w}([0, T] ; W) \cap C_{w}^{1}([0, T] ; X),
$$

where the subscript $w$ of $C_{w}$ and $C_{w}^{1}$ indicates the weak continuity. Moreover, $\sup _{0<t<T}\|u(t)\|_{W}$ depends only on $T, \beta$, and $\|u(0)\|_{W}$.

This theorem is not concerned with the uniqueness of the solution. Neither is it concerned with whether the weak continuity can be strong continuity. However, these two issues are settled rather straightforwardly in individual cases of applications.

With the theorem above, we may prove the local existence (Theorem 3.1) in the following way. We define

$$
A(\omega)=a v \omega_{x}-v_{x} \omega .
$$

For $\omega \in W=H^{1}\left(S^{1}\right) / \mathbb{R}$, we have $v \in V$. Therefore Sobolev's inequality implies that

$$
\begin{aligned}
\|A(\omega)\| & \leq|a|\|v\|_{\infty}\left\|\omega_{x}\right\|+\|H \omega\|\|\omega\|_{\infty} \leq c_{0}|a|\left\|v_{x}\right\|\left\|\omega_{x}\right\|+c_{0}\|\omega\|\left\|\omega_{x}\right\| \\
& =c_{0}(|a|+1)\|\omega\|\left\|\omega_{x}\right\| .
\end{aligned}
$$

Similarly we have

$$
\|A(\omega)-A(\zeta)\| \leq C(1+|a|)\left(\left\|\omega_{x}\right\|+\left\|\zeta_{x}\right\|\right)\left\|\omega_{x}-\zeta_{x}\right\| .
$$

This shows that $A: W \rightarrow X$ is strongly continuous. We then consider

$$
\langle\omega, A(\omega)\rangle=\left(\omega_{x}, A(\omega)_{x}\right)=\left(\frac{a}{2}-1\right) \int_{-\pi}^{\pi} v_{x}(t, x) \omega_{x}(t, x)^{2} \mathrm{~d} x-\int_{-\pi}^{\pi} \omega \omega_{x} H \omega_{x} \mathrm{~d} x .(12)
$$

By (6) we have

$$
|\langle\omega, A(\omega)\rangle| \leq C(1+|a|)\left\|\omega_{x}\right\|^{3}
$$

with an absolute constant $C$. Therefore (11) is satisfied with $\beta(r)=C(1+|a|) r^{3 / 2}$, which completes the proof of the existence of a solution.

Uniqueness of the solution is proved in the usual way. Let $\omega$ and $\zeta$ be a solution for the same initial data. Then

$$
\omega_{t}-\zeta_{t}=-a v(\omega-\zeta)_{x}-a(v-u) \zeta_{x}+v_{x}(\omega-\zeta)+(v-u)_{x} \zeta
$$


where $v_{x}=H \omega$ and $u_{x}=H \zeta$. Taking an $L^{2}$ inner-product with $\omega-\zeta$, we have

$$
\begin{aligned}
\frac{1}{2} \frac{\mathrm{d}}{\mathrm{d} t}\|\omega(t)-\zeta(t)\|^{2}= & \frac{2+a}{2} \int_{-\pi}^{\pi} v_{x}(\omega(t)-\zeta(t))^{2} \mathrm{~d} x \\
& +\int_{-\pi}^{\pi}\left[\zeta(v-u)_{x}(\omega-\zeta)-a \zeta_{x}(v-u)(\omega-\zeta)\right] \mathrm{d} x \\
\leq & \frac{2+|a|}{2}\left\|v_{x}\right\|_{\infty}\|\omega(t)-\zeta(t)\|^{2}+\|\zeta\|_{\infty}\left\|v_{x}-u_{x}\right\|\|\omega-\zeta\| \\
& +|a|\left\|\zeta_{x}\right\|\|v-u\|_{\infty}\|\omega-\zeta\| \\
\leq & C(1+|a|) M\|\omega(t)-\zeta(t)\|^{2}
\end{aligned}
$$

where $M=\max _{0 \leq t \leq T}\left(\left\|\omega_{x}(t)\right\|+\left\|\zeta_{x}(t)\right\|\right)$. Uniqueness follows from this.

The strong continuity of $t \mapsto \omega(t)$ is proved just in the same way as in [10], see page 23 of [10]. We thus obtain Theorem 3.1.

Proof of Theorem 3.2: In view of Theorem 3.1, it is sufficient to prove that the $H^{1}$ norm of $\omega(t)$ remains bounded as $t \rightarrow T$. The equation (12) shows that

$$
\frac{1}{2} \frac{\mathrm{d}}{\mathrm{d} t}\left\|\omega_{x}(t)\right\|^{2}=\frac{2-a}{2} \int_{-\pi}^{\pi} \omega_{x}(t)^{2} H \omega(t) \mathrm{d} x+\int_{-\pi}^{\pi} \omega(t) \omega_{x}(t) H \omega_{x}(t) \mathrm{d} x .
$$

Note that

$$
\int_{-\pi}^{\pi} \omega \omega_{x} H \omega_{x} \mathrm{~d} x=\int_{-\pi}^{\pi} H \omega \cdot H\left(\omega_{x} H \omega_{x}\right) \mathrm{d} x .
$$

Since $H\left(\omega_{x} H \omega_{x}\right)=-\frac{1}{2}\left(\omega_{x}^{2}-\left(H \omega_{x}\right)^{2}\right)$, we have

$$
\int_{-\pi}^{\pi} \omega \omega_{x} H \omega_{x} \mathrm{~d} x=-\frac{1}{2} \int_{-\pi}^{\pi} H \omega \cdot\left(\left(\omega_{x}\right)^{2}-\left(H \omega_{x}\right)^{2}\right) \mathrm{d} x .
$$

Summing up these equalities, we obtain

$$
\begin{aligned}
\frac{1}{2} \frac{\mathrm{d}}{\mathrm{d} t}\left\|\omega_{x}(t)\right\|^{2} & =\frac{1-a}{2} \int_{-\pi}^{\pi} \omega_{x}(t)^{2} H \omega(t) \mathrm{d} x+\frac{1}{2} \int_{-\pi}^{\pi} H \omega\left(H \omega_{x}\right)^{2} \mathrm{~d} x \\
& \leq \frac{|a-1|}{2}\|H \omega(t)\|_{\infty}\left\|\omega_{x}(t)\right\|^{2}+\frac{1}{2}\|H \omega(t)\|_{\infty}\left\|H \omega_{x}(t)\right\|^{2} \\
& =\frac{|a-1|}{2}\|H \omega(t)\|_{\infty}\left\|\omega_{x}(t)\right\|^{2}+\frac{1}{2}\|H \omega(t)\|_{\infty}\left\|\omega_{x}(t)\right\|^{2},
\end{aligned}
$$

which is written as

$$
\frac{\mathrm{d}}{\mathrm{d} t}\left\|\omega_{x}(t)\right\|^{2} \leq(|a-1|+1)\|H \omega(t)\|_{\infty}\left\|\omega_{x}(t)\right\|^{2} .
$$

By Gronwall's inequality, we have

$$
\left\|\omega_{x}(t)\right\|^{2} \leq\left\|\omega_{x}(0)\right\|^{2} \exp \left((|a-1|+1) \int_{0}^{t}\|H \omega(s)\|_{\infty} \mathrm{d} s\right) .
$$

Namely, $\left\|\omega_{x}(t)\right\|$ remains bounded if

$$
\int_{0}^{T}\|H \omega(t)\|_{\infty} \mathrm{d} t<\infty
$$

This ends the proof of Theorem 3.2. 
We finally prove a proposition on further regularity of solutions.

Proposition 5.1 Let $m$ be an integer $\geq 2$. If $\omega_{0} \in H^{m}\left(S^{1}\right) / \mathbb{R}$, then $\sup _{0 \leq t \leq T}\|\omega(t)\|_{H^{m}}<\infty$ as far as the solution $\omega$ exists in $C\left([0, T] ; H^{1}\right)$.

Proof. We prove in the case of $m=2$. Other cases are proved similarly. We note first that

$$
\omega_{t x x}=-a v \omega_{x x x}+(1-2 a) v_{x} \omega_{x x}+(2-a) v_{x x} \omega_{x}+v_{x x x} \omega
$$

This yields

$$
\frac{1}{2} \frac{\mathrm{d}}{\mathrm{d} t}\left\|\omega_{x x}(t)\right\|^{2}=\frac{2-3 a}{2} \int_{-\pi}^{\pi} v_{x} \omega_{x x}^{2}+(2-a) \int_{-\pi}^{\pi} v_{x x} \omega_{x} \omega_{x x}+\int_{-\pi}^{\pi} v_{x x x} \omega \omega_{x x} .
$$

The first integral of the right hand side is bounded by $\|H \omega\|_{\infty}\left\|\omega_{x x}(t)\right\|^{2}$, the third by $\|\omega(t)\|_{\infty}\left\|H \omega_{x x}(t)\right\|\left\|\omega_{x x}(t)\right\|$. Both are further bounded by a constant multiple of $\left\|\omega_{x}(t)\right\|\left\|\omega_{x x}(t)\right\|^{2}$. The second integral is bounded as

$$
\int_{-\pi}^{\pi} v_{x x} \omega_{x} \omega_{x x} \leq\left\|H \omega_{x}\right\|_{L^{4}}\left\|\omega_{x}\right\|_{L^{4}}\left\|\omega_{x x}\right\| \leq c\left\|\omega_{x}\right\|_{L^{4}}^{2}\left\|\omega_{x x}\right\|
$$

since the Hilbert transform is a bounded operator in $L^{4}$ (see, e.g., [11] or [19]). We now use the following Gagliardo-Nirenberg inequality (see, 139 page of [1]):

$$
\|f\|_{L^{4}} \leq c\|f\|^{3 / 4}\left\|f_{x}\right\|^{1 / 4} \quad\left(f \in H^{1}(-\pi, \pi)\right) .
$$

The last term of (13) is now bounded by $\left\|\omega_{x}\right\|^{3 / 2}\left\|\omega_{x x}\right\|^{3 / 2} \leq c\left\|\omega_{x}\right\|\left\|\omega_{x x}\right\|^{2}$.

Summing up these inequalities, we have

$$
\frac{1}{2} \frac{\mathrm{d}}{\mathrm{d} t}\left\|\omega_{x x}(t)\right\|^{2} \leq C(1+|a|)\left\|\omega_{x}(t)\right\|\left\|\omega_{x x}(t)\right\|^{2} .
$$

Gronwall's inequality yields

$$
\left\|\omega_{x x}(t)\right\|^{2} \leq\left\|\omega_{x x}(0)\right\|^{2} \exp \left(2 C(1+|a|) \int_{0}^{t}\left\|\omega_{x}(s)\right\| \mathrm{d} s\right) .
$$

We have already proved that the integral on the right hand side is bounded by a certain function of $\|H \omega\|_{\infty}$. Therefore the boundedness of $\omega_{x x}(t)$ is proved.

Remark 5.1 Note that $\omega_{x}(t)$ is bounded by an exponential function of $\int_{0}^{t}\|H \omega\|_{\infty}$, and $\omega_{x x}(t)$ is bounded double exponentially. It can therefore be quite large for a relatively small $t$. 


\section{The case of $a=\infty$}

If we set $\omega=a^{-1} \tilde{\omega}$ in (3), and if we multiply the resultant equation by $a$ and let $a \rightarrow \infty$, then, after deleting the tilde, we have

$$
\omega_{t}+v \omega_{x}=0, \quad v_{x}=H \omega .
$$

We consider this equation with the initial condition $\omega(0, x)=\omega_{0}(x)$. Although De Gregorio's equation is a model for the 3D Euler equations, the equation (14) has a similarity with the $2 \mathrm{D}$ Euler equations in vorticity form, as we will see in what follows. We now prove

Theorem 6.1 Suppose that $\omega_{0}$ belongs to $H^{1}\left(S^{1}\right) / \mathbb{R}$. Then the solution of (14) with $\omega(0)=\omega_{0}$ exists for $0 \leq t<\infty$.

Proof. Suppose that $\omega_{0} \in H^{1}\left(S^{1}\right) / \mathbb{R}$. The proof of the local existence for (3) is still applicable in the present equation, and we have a local solution. An analogue of Theorem 3.2 is also proved in the same way, and we have a global solution if $\int_{0}^{T}\|H \omega(t)\|_{\infty} \mathrm{d} t<\infty$ for any $T>0$.

Suppose now that the solution of

$$
\omega_{t}+v \omega_{x}=0, \quad v=-\left(-\frac{\mathrm{d}^{2}}{\mathrm{~d} x^{2}}\right)^{-1 / 2} \omega
$$

exists in $0 \leq t \leq T$, and set $M=\sup _{0 \leq t \leq T}\left\|\omega_{x}(t)\right\|$. Note that $\omega$ is represented as

$$
\omega\left(t, X_{t}(x)\right)=\omega_{0}(x)
$$

where $X_{t}(x)$ is a solution of

$$
\frac{\mathrm{d}}{\mathrm{d} t} X_{t}=v\left(t, X_{t}(\xi)\right), \quad X_{0}(\xi)=\xi
$$

Sobolev's embedding theorem implies that $H^{1}\left(S^{1}\right) \subset C^{1 / 2}\left(S^{1}\right) . \omega(t)$ is therefore a $\frac{1}{2}$-Hölder continuous function. Note also that the Hilbert transform is a bounded operator in the Hölder class ([19, page 121]). Consequently,

$$
\|v(t)\|_{C^{1,1 / 2}} \leq C\|\omega(t)\|_{C^{1 / 2}} \leq C^{\prime} M \quad(0 \leq t \leq T) .
$$

In particular, the Lipschitz norm of $v(t)$ is bounded in $t$. Therefore the ordinary differential equation (16) has a solution which is unique with respect to the initial datum $\xi \in[0,2 \pi]$. As an immediate consequence of (15), we have

$$
\|\omega(t)\|_{\infty}=\left\|\omega_{0}\right\|_{\infty} .
$$

We next prove that

$$
|v(t, x)-v(t, y)| \leq G(|x-y|) \quad(x, y \in[0,2 \pi]),
$$

where $G$ is defined by

$$
G(s)=C\left\|\omega_{0}\right\|_{\infty} \times \begin{cases}s(1-\log s) & (0 \leq s \leq 1) \\ 1 & (1<s)\end{cases}
$$


with an absolute constant $C$. The inequality (18) can be proved by

$$
v(t, x)=\frac{1}{\pi} \int_{-\pi}^{\pi} \omega(t, y) \log \left|\sin \frac{x-y}{2}\right| \mathrm{d} y .
$$

Let $\delta=|x-y|$. We do not lose generality if we assume that $\delta<1$ and $0<x<y<2 \pi$. We have

$$
v(t, x)-v(t, y)=\frac{1}{\pi} \int_{-\pi}^{\pi} \omega(t, z)\left(\log \left|\sin \frac{x-z}{2}\right|-\log \left|\sin \frac{y-z}{2}\right|\right) \mathrm{d} z .
$$

The domain of integration is divided into $0<z<x-\delta / 2, x-\delta / 2<z<$ $x+\delta / 2, x+\delta / 2<z<y+\delta / 2, y+\delta / 2<z<2 \pi$. In each subinterval $\omega$ is bounded by $\left\|\omega_{0}\right\|_{\infty}$, and the necessary inequalities are derived as is common in the potential theory. We prove only one case.

$$
\begin{aligned}
\int_{x-\delta / 2}^{x+\delta / 2} & \omega(t, z)\left(\log \left|\sin \frac{x-z}{2}\right|-\log \left|\sin \frac{y-z}{2}\right|\right) \mathrm{d} z \\
\leq & \left\|\omega_{0}\right\|_{\infty} \int_{-\delta / 2}^{\delta / 2}\left(|\log | \sin \frac{z}{2}||+|\log | \sin \frac{y-x-z}{2}||\right) \mathrm{d} z \\
\leq & c\left\|\omega_{0}\right\|_{\infty} \delta(1+|\log \delta|) .
\end{aligned}
$$

Since the Hilbert transform is a bounded operator in the Hölder class, we see for $\beta \in(0,1)$ that

$$
\|H \omega(t)\|_{\infty} \leq c_{1}\|H \omega(t)\|_{C^{\beta}} \leq c_{2}\|\omega(t)\|_{C^{\beta}},
$$

where $c_{1}$ and $c_{2}$ depend only on $\beta$. Therefore it is enough to show that for any $T>0$ there exists a $\beta \in(0,1)$ such that $\sup _{0<t<T}\|\omega(t)\|_{C^{\beta}}<\infty$. Since

$$
|\omega(t, x)-\omega(t, y)| \leq c|| \omega_{0, x} \|\left|X_{t}^{-1}(x)-X_{t}^{-1}(y)\right|^{1 / 2},
$$

we must derive an a priori bound on $\left|X_{t}^{-1}(x)-X_{t}^{-1}(y)\right|$.

Let us write $q(t, x)=X_{t}^{-1}(x)$. It is then characterized by

$$
\frac{\partial}{\partial t} q(t, x)=-v(t, q(t, x)), \quad q(0, x)=x .
$$

This equation and (18) give us

$$
\frac{\partial}{\partial t}|q(t, x)-q(t, y)| \leq G(|q(t, x)-q(t, y)|) .
$$

It is known that this differential inequality can be solved. In fact, define $\beta(t)$ by $\beta(t)=\exp \left(-C\left\|\omega_{0}\right\|_{\infty} t\right)$. Define also

$$
z(t)=|x-y|^{\beta(t)} \exp (1-1 / \beta(t))
$$

for $t$ such that the right hand side is less than one, and

$$
z(t)=1+C\left\|\omega_{0}\right\|_{\infty}\left(t-t_{0}\right)
$$


for later $t$ with $t_{0}$ being the time when the right hand side of (22) becomes one. We then have (see, for instance, [13, page 73])

$$
|q(t, x)-q(t, y)| \leq z(t) .
$$

By (20) and (21) the proof is complete.

\section{Concluding remarks}

The above proof depends on the fact that a solution of the ODE (16) exists uniquely and estimated only by $\|\omega\|_{\infty}$, which is guaranteed by (15). If $a$ is finite, then we do not have means to find an a priori bound of $\|\omega(t)\|_{\infty}$. Accordingly, the proof above does not seem to be applicable to the case of finite $a$.

By Theorem 6.1 together with the results in $[4,5,6]$, one may be tempted to conjecture that solutions may blow-up for $-1 \leq a<1$, and they exist globally for $-\infty<a<-1$ and $1 \leq a<\infty$. We tested this conjecture by numerical experiments, the results of which will be reported elsewhere.

Finally, some potentially useful facts are collected here.

Proposition 7.1 If $a=1$, and if the solution is odd in $x$, then $\omega_{x}(t, 0) \equiv \omega_{0, x}(0)$.

This is Proposition 3 of [18]. The proof is easy: By differentiation, we have

$$
\omega_{t x}=-v \omega_{x x}+v_{x x} \omega
$$

The right hand side vanishes at $x=0$ because of the oddness.

Proposition 7.2 If $-\infty<a<-1$, then

$$
\|\omega(t)\|_{L^{p}}=\left\|\omega_{0}\right\|_{L^{p}},
$$

where $p=-a$.

The proof is straightforward.

$$
\begin{aligned}
\frac{\mathrm{d}}{\mathrm{d} t} \int_{-\pi}^{\pi}|\omega(t, x)|^{p} \mathrm{~d} x & =p \int_{-\pi}^{\pi}|\omega(t, x)|^{p-2} \omega(t, x) \omega_{t}(t, x) \mathrm{d} x \\
& =p \int_{-\pi}^{\pi}|\omega|^{p-2}\left(-a v \omega \omega_{x}+v_{x} \omega^{2}\right) \mathrm{d} x \\
& =-a \int_{-\pi}^{\pi} v\left(|\omega|^{p}\right)_{x} \mathrm{~d} x+p \int_{-\pi}^{\pi} v_{x}|\omega|^{p} \mathrm{~d} x \\
& =0 .
\end{aligned}
$$




\section{References}

[1] Adams R A and Fournier J J F 2003 Sobolev Spaces, 2nd ed. (Amsterdam: Academic Press)

[2] Beale J T, Kato T and Majda A 1984 Remarks on the breakdown of smooth solutions for the 3-D Euler equations Comm. Math. Phys. 9466

[3] Chae D, Córdoba A, Córdoba D and Fontelos M A 2005 Finite time singularities in a 1D model of the quasi-geostrophic equation Adv, Math. 194203

[4] Córdoba A, Córdoba D and Fontelos M A, 2006 Integral inequalities for the Hilbert transform applied to a nonlocal transport equation J. Math. Pure Appl. 86529

[5] Córdoba A, Córdoba D and Fontelos M A, 2005 Formation of singularities for a transport equation with nonlocal velocity Ann. Math. 1621

[6] Constantin P, Lax P D and Majda A J 1985 A simple one-dimensional model for the threedimensional vorticity equation Comm. Pure Appl. Math. 38715

[7] De Gregorio S 1990 On a one-dimensional model for the three-dimensional vorticity equation $J$. Stat. Phys. 591251

[8] De Gregorio S 1996 A partial differential equation arising in a 1D model for the 3D vorticity equation Math. Meth. Appl. Sci. 191233

[9] Hou T Y and Lei Z, 2008 On the stabilizing effect of convection in three-dimensional incompressible flows Comm. Pure. Appl. Math. doi: 10.1002/cpa.20254

[10] Kato T and Lai C Y 1984 Nonlinear evolution equations and the Euler flow J. Func. Anal. 5615

[11] Katznelson Y 2003 An Introduction to Harmonic Analysis 3rd ed. (Cambridge:Cambridge University Press)

[12] Krasny R 1986 A study of singularity formation in a vortex sheet by the point-vortex approximation J. Fluid Mech., $\mathbf{1 6 7} 65$

[13] Marchioro C and Pulvirenti M 1994 Mathematical Theory of Incompressible Nonviscous Fluids (New York: Springer-Verlag)

[14] Morlet A C 1998 Further properties of a continuum of model equations with globally defined flux J. Math. Anal. Appl. 221132

[15] Okamoto H Well-posedness of the generalized Proudman-Johnson equation without viscosity, to appear in J. Math. Fluid Mech.

[16] Okamoto H and Ohkitani K 2005 On the role of the convection term in the equations of motion of incompressible fluid J. Phys. Soc. Japan 742737

[17] Okamoto H and Zhu J 2000 Some similarity solutions of the Navier-Stokes equations and related topics Taiwanese J. Math. 465

[18] Sato H and Sakajo T 2006 Numerical study of De Gregorio's model for the 3D vorticity equation Trans. Japan SIAM 16221 (in Japanese)

[19] Zygmund A 2002 Trigonometric Series, 3rd ed. (Cambridge: Cambridge University Press) 